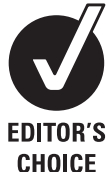

'Department of Neurologic Surgery, The Mayo Clinic, Rochester, Minnesota, USA ${ }^{2}$ Department of Neurosurgery, Massachusetts General Hospital, Harvard Medical School, Boston, Massachusetts, USA

${ }^{3}$ Division of Neurosurgery, University of California, San Diego, California, USA ${ }^{4}$ Department of Neurosurgery, Beth Israel Deaconess Hospital, Harvard Medical School, Boston, Massachusetts, USA ${ }^{5}$ Section of Neurosurgery, The University of Chicago, Chicago, Illinois, USA

\section{Correspondence to} Dr Clark C Chen, Department of Neurosurgery, Beth Israel Deaconess Hospital/Harvard Medical School, Dana-Farber Cancer Institute, Jimmy Fund 620A, 44 Binney Street, Boston, MA 02115-6084, USA: cchen1@partners.org

Received 12 October 2010 Revised 30 March 2011 Accepted 7 April 2011 Published Online First 20 May 2011

\title{
A review of lumbar spinal instrumentation: evidence and controversy
}

\author{
Maya A Babu, ${ }^{1}$ Jean-Valery C Coumans, ${ }^{2}$ Bob S Carter, ${ }^{3}$ William R Taylor, ${ }^{3}$ \\ Ekkehard M Kasper, ${ }^{4}$ Ben Z Roitberg, ${ }^{5}$ William E Krauss, ${ }^{1}$ Clark C Chen ${ }^{4}$
}

\section{ABSTRACT}

Disability secondary to disorders of the spine is a significant problem worldwide. In the USA, there has been a recent surge in the costs associated with caring for spinal pathology; from 1997 to 2005, there was a growth of $65 \%$ in healthcare expenditures on spinal disease, totalling $\$ 86$ billion in 2005. Increasingly, there has been media and public scrutiny over the rapid rise in the volume of procedures with spinal instrumentation; some have suggested that this rise has been fuelled by non-medical drivers such as the financial incentives involved with the use of instrumentation; others suggest that innovation in spine technology and devices has led to improved options for the treatment of spine pathology. In this context, we conducted a review of the literature to assess the use of instrumentation in lumbar procedures and its relationship to successful fusion and patient outcome. Our review suggests that there is data supporting the thesis that lumbar instrumentation improves rates of fusion. However, there is no consistent correlation between increased rates of fusion and improved patient outcomes.

\section{INTRODUCTION}

The leading cause of disability in the USA involves diseases of the musculoskeletal system. Of these disorders, those affecting the spine most commonly require medical or surgical care. ${ }^{1}$ Interestingly, healthcare expenditures related to disorders of the spine have grown 65\% between 1997 and 2005, totalling $\$ 86$ billion in 2005 . $^{2}$ In the USA, this rapid rise in healthcare expenditures has prompted media and Congressional scrutiny of the drivers behind this escalation. Specifically, much attention recently has been paid to the increased use of instrumented spinal fusion. Consider the following excerpt from the 11 September 2010 New York Times editorial on the topic of instrumented spinal instrumentation:

[There is a rapid rise in] the use of this surgery, which fuses multiple discs in the spine, in patients who would have done better, and faced fewer risks, with simpler surgery that eases pressure on the nerves without fusion... The explanation for the boom was likely economic. Surgeons were paid 10 times as much for the complex surgery, hospitals were paid three and a half times as much, and manufacturers reaped a bonanza selling $\$ 50000$ worth of implants for the complex surgery, compared with little or no profit from the simpler surgery. ${ }^{3}$

Spine fusion is a surgical technique used to induce bone formation between adjacent vertebrae.
In doing so, the adjacent vertebrae become fused and function as a single mechanical unit. This fusion is achieved by decorticating opposing bone surfaces and packing the gap between these surfaces with bone-graft material. Most frequently, the fusion is accompanied by the insertion of rigid implants that serve to minimise motion between the opposing bone surfaces. This practice is known as spinal instrumentation. The basic principle behind spinal instrumentation is built upon the observation that movement of opposing fractured bone surfaces impairs bone fusion and increases the likelihood of non-union. By connecting the implants in ways that augment the stability of the spine, it is thought that instrumentation functions as an 'internal' brace to facilitate bony fusion. ${ }^{4}$

While instrumented spine fusion was initially developed for the treatment of conditions associated with a grossly unstable spine ${ }^{5-7}$ including traumatic/pathological fractures and deformity correction, these indications now account for a small fraction of the procedures performed. ${ }^{8}$ The most common indication for spinal instrumentation is now degenerative spinal disorders without gross mechanical instability. This expansion in surgical indication has led to a significant rise in the frequency of spinal fusions. Over the period spanning 2002 to 2007, there was an approximate 15 -fold increase in lumbar instrumentation in Medicare recipients. 9

It is possible that the increased utilisation of spinal fusion reflects improved quality in care for an ageing population. Technological advances in spine instrumentation and imaging have certainly contributed to this increase. ${ }^{9-11}$ However, some have linked potential financial incentives to increased procedure utilisation. On average, a hospital bills more than $\$ 34000$ per instrumented fusion, excluding professional fees. ${ }^{8}$ The market for spinal implants is estimated to be $\$ 3.7$ billion in 2008 , with a projected annual growth rate of $18-20 \% .^{12} 13$

Adding to this controversy is a lack of general consensus in the neurosurgical and orthopaedic communities as to the indications for spinal fusion, particularly in the treatment of degenerative lumbar spine disorders. While guidelines have been published by various professional societies, these guidelines remain vague and subject to a wide range of interpretations. Importantly, despite significant regional variation in the frequency of spinal fusion performed in the USA, there is no evidence of differences in quality between regions with varying fusion frequency. ${ }^{14}$ 
SPINAL INSTRUMENTATION: BACKGROUND AND RATIONALE Spinal instrumentation was initially developed in the 1950s as a treatment for paediatric populations afflicted with polio and subsequent spinal deformity. The deformity was corrected by surgically destabilising the spine in a way that would allow for correction. The instrumentation was implanted to maintain the corrected alignment while facilitating fusion across the destabilised segments. The most famous and successful construct developed in this period was the Harrington Rod, a simple distraction device where rods are secured to the spine at two ends using hooks (figure 1A). ${ }^{15} 16$

The spectrum of instrumentation design has expanded greatly since the development of the Harrington Rod. Despite the multitude of instrument designs, instrumentation can be conceptualised within two categories: rigid and non-rigid. Rigid devices include segmental systems and interbody devices, such as cages. Non-rigid devices include prostheses such as artificial discs. Since non-rigid devices are generally designed for motion preservation and not used in conjunction with fusion, the remainder of the discussion will focus on rigid devices.

Rigid spinal implants are generally divided into two types: segmental devices and interbody devices. Segmental systems utilise instruments that are fixed onto each individual vertebra. The instruments are then connected in a rigid manner. The pedicle screw (figure 1B) is an example of this type. In this design, screws are placed into the vertebral bodies and connected by a rigid construct (a plate or a rod) thereby immobilising the spanning segments. Interbody devices utilise constructs to span a discontinuous bony surface. The intervertebral cage is an example of this design (figure 1C). The cage is frequently packed with autologous bone and inserted into fracture or discectomy sites to maintain spinal alignment. Each type of instrumentation has been used alone or in combination, depending on the instrumented level and anatomical considerations.

Instrumented spinal fusion in the lumbar region is typically performed when back pain is thought to be related to: (1) abnormal motion between lumbar vertebrae, (2) aberrant spinal alignment such as spondylolisthesis (anterior subluxation of one lumbar vertebrae on another) or (3) painful motion segment which can be discogenic, facet mediated, or both. It is thought that elimination of these 'pain generators' by spinal fusion would afford symptomatic relief. However, the determination of spinal instability, aberrant spinal alignment, or painful disc/facet disruption is highly dependent on the evaluating surgeon. For instance, some surgeons will insist on radiographic demonstration of gross movement on flexion and extension, radiographic evidence of nerve root compression with corroborating neurological findings, and back pain before considering surgery. Others may require only a clinical history of back pain that worsens

with physical motion. There is little or no consensus among practitioners in terms of surgical indication for instrumented spinal fusion.

\section{EFFICACY OF SPINAL FUSION: A CENTRAL OUESTION}

Fusion in the lumbar spine can be performed using a number of different techniques. However, if one accepts the premise that the purpose of rigid instrumentation is to facilitate the rate of bony fusion, the specifics of how the fusion is achieved (ie, what techniques were used to facilitate fusion) are then less pertinent than the question of whether successful fusion is correlated with improved clinical outcome. We examined this central question using the available data for lumbar instrumented postero-lateral fusion (defined below). We selected this dataset because of the availability of multiple, well-designed randomised controlled trials (class Ib data).

Postero-lateral fusion refers to the surgical technique whereby bone grafts are placed in between decorticated bony surfaces of the transverse processes (figure 1B) and, if feasible, the facet joints. Importantly, postero-lateral fusion can be achieved without spinal instrumentation. However, in recent years, postero-lateral fusion has been accompanied by segmental instrumentation (with screws placed through the pedicle and into the vertebral body and connected by rigid rods). The instrumentation is thought to immobilise the connected vertebrae and facilitate bony fusion. Analysis of randomised trials comparing non-instrumented and instrumented postero-lateral fusion, thus, affords one the opportunity to study whether spinal instrumentation affected clinical outcome in patients who essentially underwent comparable surgeries.

\section{METHODS}

We performed a search of the PubMed database and CENTRAL (The Cochrane Library 2008, Issue 2) for literature published from 1966 to 2010 using key words and MeSH headings including 'lumbar fusion,' 'outcome,' 'surgery,' 'simple,' 'instrumentation,' and 'complex.' The search was restricted to the English language and yielded 318 references. The title and abstracts of each of these references were reviewed, and papers not providing class Ib data comparing non-instrumented and instrumented lumbar fusion were discarded. In the end, we identified seven well-designed randomised controlled trials (class Ib data).

\section{RESULTS}

Of the seven studies, four concluded that segmental instrumentation did not affect the likelihood of successful fusion or clinical outcome $e^{17-20}$ (table 1); three studies provided data that

Figure 1 Illustrations of spinal instrumentation. (A). Harrington Rod. Left: schematic of a Harrington Rod; middle: photograph of a Harrington Rod; right: x-ray (lateral view) of an implanted Harrington Rod. (B). Pedicle screws. Left top: schematic of a pedicle screw; left bottom: photograph of pedicle screws placed at $\mathrm{L} 3, \mathrm{~L} 4$ and $\mathrm{L} 5$, connected with a rigid rod. The white rectangular region denotes where bone grafts are placed for postero-lateral fusions. (C). Intervertebral cage construct. Left: photograph of

a intervertebral cage construct; right: $x$-ray (lateral view) of an implanted cage construct.
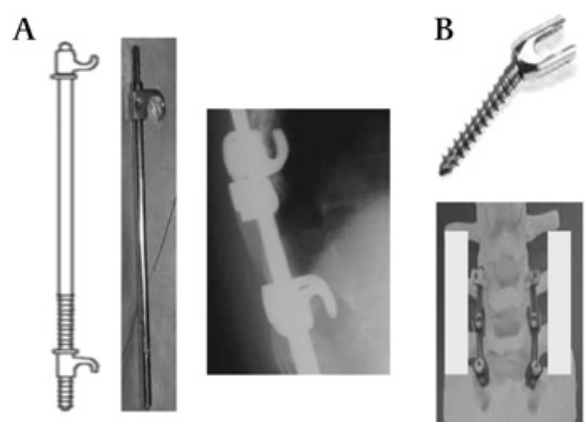

C

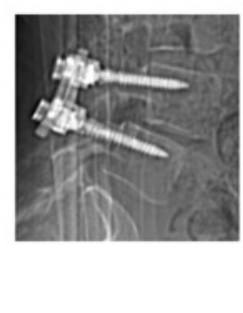

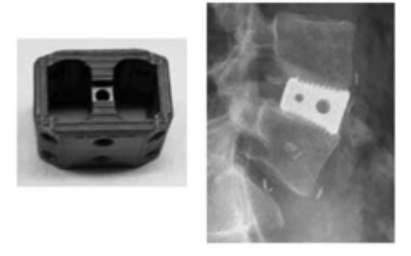


Table 1 Four studies reviewed that showed equivocal findings

\begin{tabular}{|c|c|c|c|c|}
\hline Author(s) (year) & Design & No of patients & Outcome assessment & Findings \\
\hline France $(1999)^{17}$ & Prospective randomised & 71 & Visual assessment score & $\begin{array}{l}\text { - No statistical difference in radiographic fusion } \\
\text { - No statistical difference in outcome measure }\end{array}$ \\
\hline Bjarke Christensen $(2002)^{18}$ & Prospective randomised & 129 & $\begin{array}{l}\text { Dallas Pain Questionnaire } \\
\text { Low-back pain rating scale } \\
\text { Assessment of work status }\end{array}$ & $\begin{array}{l}\text { - No statistical difference in radiographic fusion } \\
\text { - No statistical difference in outcome measure }\end{array}$ \\
\hline Moller $(2000)^{19}$ & Prospective randomised & 111 & Visual assessment score & $\begin{array}{l}\text { - No statistical difference in radiographic fusion } \\
\text { - No statistical difference in outcome measure }\end{array}$ \\
\hline Thomsen $(1997)^{20}$ & Prospective randomised & 130 & Dallas Pain Questionnaire & $\begin{array}{l}\text { - No statistical difference in radiographic fusion } \\
\text { - No statistical difference in outcome measure }\end{array}$ \\
\hline
\end{tabular}

segmental instrumentation enhanced the rate of successful fusion. We reviewed the latter studies to determine whether the increased fusion rate corresponds to improved clinical outcome $e^{21-23}$ (table 2).

Zdeblick $^{21}$ randomised 124 patients with lower-back pain attributable to aberrant lumbar motion. Patients were randomised into groups that underwent postero-lateral onlay fusion or instrumented fusion. Patients with osteoporosis were moved from the instrumentation group to the non-instrumentation group owing to concerns that osteoporosis impairs pedicle screw fixation. Clinical outcome was assessed by patient report using a scale of excellent, good, fair and poor. Follow-up was 9-28 months. Fusion occurred in $65 \%$ of non-instrumented patients and $95 \%$ of the instrumented patients $(p=0.002)$. Forty-nine per cent of the non-instrumented patients reported an excellent outcome, while $70 \%$ of the instrumented patients reported an excellent outcome.

A second study by Fritzell et $a^{22}$ randomised 294 patients to postero-lateral onlay fusion versus different types of instrumentation supplemented with fusion, including pedicle screw. Ninety-eight per cent of the patients were followed for 2 years. The clinical outcome was assessed by the Oswestry Disability Index, General Function Score and Visual Analogue Scale (for pain assessment). The fusion rates for the on-lay fusion and pedicle screw group were $72 \%$ and $87 \%$, respectively $(p=0.004)$. There was no significant difference in any of the outcome scores between the groups, whether or not they fused.

A third study by Fischgrund et $a l^{23}$ randomised 76 symptomatic spondylolisthesis patients to postero-lateral onlay fusion or instrumented fusion. The clinical outcome was assessed using patient satisfaction surveys (graded as excellent, good and poor). The 2-year follow-up was obtained in $88 \%$ of the patients. Successful fusion was achieved in $82 \%$ of the instrumented patients and $45 \%$ of the non-instrumented patients $(p=0.0015)$. However, the occurrence of successful fusion did not correlate with clinical outcome. Outcome was graded as excellent or good in $76 \%$ of the instrumented patients and $85 \%$ of the non-instrumented patients.

These studies suggest that the successful fusion rate for lumbar non-instrumented fusion ranged from $45 \%$ to $72 \%$.
Between $15 \%$ and $38 \%$ of the patients benefitted from instrumentation in terms of achieving fusion. However, the enhancement in bony fusion did not necessarily translate into improved clinical outcomes. Two of the three studies reviewed showed that an improved fusion rate related to spinal instrumentation did not correlate with clinical outcome. While the Zdeblick study demonstrated that instrumentation improved both fusion rate and clinical outcome, there is a noticeable discrepancy in the magnitude of these respective improvements. In this context, the data suggest a poor correlation between successful fusion and improved clinical outcome.

\section{DISCUSSION AND CONCLUSION}

Extrapolating results from the various studies reviewed and offering one coherent clinical thesis is difficult. From a study design perspective, there was significant variation across the publications reviewed in terms of indications, inclusion and exclusion criteria, duration from the onset of symptoms to the time of surgery, methods of outcome assessment, and the level of supportive care provided to patients. For instance, only one $e^{22}$ of the studies reviewed here examined the effect of instrumentation on low-back pain without laminectomy, a procedure that would further destabilise the spine. All other studies included variable number of patients who underwent laminectomy in addition to either instrumented or non-instrumented fusion. As another example, two of the studies ${ }^{22} 23$ included only patients who did not undergo prior surgery whereas other studies contained variable numbers of patients with previously failed back surgery.

From a surgical perspective, differences in surgical expertise, technique and instrumentation design confound any direct comparisons. From a patient selection point of view, differential society attitudes towards pain, differing practice patterns among surgeons as well as inherent genetic variations in the studied population may limit the generalisability of any particular study.

As a whole, however, the studies reviewed here suggest that there appears to be a 'disconnect' between spinal instrumentation and clinical outcome. There were four studies yielding class $\mathrm{Ib}$ data suggesting that spinal instrumentation in the lumbar

Table 2 Three studies reviewed in this paper

\begin{tabular}{|c|c|c|c|c|}
\hline Author(s) (year) & Design & No of patients & Outcome assessment & Findings \\
\hline Zdeblick $(1993)^{21}$ & Prospective randomised & 124 & Scale of excellent, good, fair and poor & $\begin{array}{l}-40 \% \text { increase in fusion with instrumentation }(p=0.002) \\
-21 \% \text { increase in rating as 'excellent outcome' with rigid } \\
\text { instrumentation }(p=0.03)\end{array}$ \\
\hline Fritzell $(2002)^{22}$ & Prospective randomised & 294 & $\begin{array}{l}\text { Oswestry Disability Score, general } \\
\text { function score, visual assessment score }\end{array}$ & $\begin{array}{l}-15 \% \text { increase in fusion with instrumentation }(p=0.004) \\
- \text { No difference in outcome assessment }\end{array}$ \\
\hline Fischgrund $(1997)^{23}$ & Prospective randomised & 76 & Scale of excellent, good and poor & $\begin{array}{l}-37 \% \text { increase in fusion with instrumentation }(p=0.0015) \\
-9 \% \text { increase in rating as 'excellent or good outcome' in } \\
\text { non-instrumented patients relative to instrumented patients }\end{array}$ \\
\hline
\end{tabular}


spine does not enhance the rate of successful bony fusion. To the extent that the ultimate goal of rigid instrumentation is to facilitate fusion, this discrepancy raises questions as to the rationale for instrumented fusion. Of three studies yielding data suggesting that instrumentation facilitates bony fusion, the improved fusion rate correlated poorly with clinical outcome. These results underscore the complexity of nociceptive transmission and higher-order processing of neural transmission. Ultimately, successful surgical treatment of degenerative spinal disorders will require a deeper understanding of the biology underlying these processes beyond the current paradigms of aberrant spinal motion, alignment or discs.

Among spine practitioners, the adaptation of new technologies seems to outpace rigorous investigations of the associated clinical efficacy. The increased use of instrumented spinal fusion occurred well before outcome data were available. Similarly, as the outcome data for instrumented fusion have just began to mature to a point where rigorous scrutiny is possible, some practitioners have already abandoned the rigid implants in favour of newer implants, including non-rigid instruments and artificial discs. If the trend of technical adaptation without convincing efficacy data continues, there is no doubt that the cost related to spinal instrumentation will continue to escalate and exacerbate the strain on the US healthcare system. On the other hand, an excessive regulation or focus on cost will necessarily stifle medical advances and compromise creativity. In the end, a balance must be achieved between these opposing forces.

\section{Competing interests None.}

Provenance and peer review Not commissioned; externally peer reviewed.

\section{REFERENCES}

1. Decade, U.S.B.a.J. The Burden of Musculoskeletal Diseases in the United States. Rosemount: The Burden of Musculoskeletal Diseases, 2008. http://www. boneandjointburden.org.

2. Martin BI, Deyo RA, Mirza SK, et al. Expenditures and health status among adults with back and neck problems. JAMA 2008;299:656-64.

3. Editorial. Is Newer Better? Not Always, in The New York Times. New York: The New York Times, 2010. p. WK10.

4. Moquin RR, Rosner MK, Cooper PB. Combined anterior-posterior fusion with laterally placed threaded interbody cages and pedicle screws for
Scheuermann kyphosis. Case report and review of the literature. Neurosurg Focus 2003;14:e10

5. Vaccaro AR, Cook CM, McCullen G. Cervical trauma: rationale for selecting the appropriate fusion technique. Orthop Clin North Am 1998;29:745-54.

6. Etame AB, Wang AC, Than $\mathrm{KD}$, et al. Outcomes after surgery for cervical spine deformity: review of the literature. Neurosurg Focus 2010;28:E14.

7. Haiyun Y, Rui G, Shucai D, et al. Three-column reconstruction through single posterior approach for the treatment of unstable thoracolumbar fracture. Spine (Phila Pa 1976) 2010;35:E295-302.

8. Quality, A.f.H.R.a. Healthcare Cost and Utilization Project. HCUPnet, Rockville, MD, USA: Agency for Healthcare Research and Quality, 2004.

9. Deyo RA, Mirza SK, Martin BI, et al. Trends, major medical complications, and charges associated with surgery for lumbar spinal stenosis in older adults. JAMA 2010;303:1259-65

10. Ciol MA, Deyo RA, Howell E, et al. An assessment of surgery for spinal stenosis: time trends, geographic variations, complications, and reoperations. J Am Geriatr Soc 1996:44:285-90

11. Silva FE, Lenke LG. Adult degenerative scoliosis: evaluation and management. Neurosurg Focus 2010;28:E1.

12. News, O.N. Spinal Industry Update. Orthop Network News 2002:13:7-8.

13. Zamanian K. Spinal Implants Market. Vancouver, BC, Canada: iData Research, 2009

14. Cook C, Santos GC, Lima R, et al. Geographic variation in lumbar fusion for degenerative disorders: 1990 to 2000. Spine J 2007:7:552-7.

15. Drummond DS. A perspective on recent trends for scoliosis correction. Clin Orthop Relat Res 1991:90-102.

16. Riebel GD, Yoo JU, Fredrickson BE, et al. Review of Harrington rod treatment of spinal trauma. Spine (Phila Pa 1976) 1993;18:479-91.

17. France JC, Yaszemski MJ, Lauerman WC, et al. A randomised prospective study of posterolateral lumbar fusion: outcomes with and without pedicle screw instrumentation. Spine (Phila Pa 1976) 1999;24:553-60.

18. Bjarke Christensen F, Stender Hansen E, Laursen M, et al. Long-term functional outcome of pedicle screw instrumentation as a support for posterolateral spinal fusion: randomized clinical study with a 5-year follow-up. Spine (Phila Pa 1976) 2002:27:1269-77.

19. Moller H, Hedlund R. Surgery versus conservative management in adult isthmic spondylolisthesis - a prospective randomized study: part 2. Spine (Phila Pa 1976) 2000;25:1711-15.

20. Thomsen K, Christensen FB, Eiskjaer SP, et al. 1997 Volvo Award winner in clinica studies. The effect of pedicle screw instrumentation on functional outcome and fusion rates in posterolateral lumbar spinal fusion: a prospective, randomized clinical study. Spine (Phila Pa 1976) 1997;22:2813-22.

21. Zdeblick TA. A prospective, randomized study of lumbar fusion. Preliminary results. Spine (Phila Pa 1976) 1993;18:983-91.

22. Fritzell $\mathbf{P}$, Hagg 0 , Wessberg $P$, et al. Chronic low back pain and fusion: a comparison of three surgical techniques: a prospective multicenter randomized study from the Swedish lumbar spine study group. Spine (Phila Pa 1976) 2002;27:1131-41.

23. Fischgrund JS, Mackay M, Herkowitz HN, et al. 1997 Volvo Award winner in clinical studies. Degenerative lumbar spondylolisthesis with spinal stenosis: a prospective, randomized study comparing decompressive laminectomy and arthrodesis with and without spinal instrumentation. Spine (Phila Pa 1976) 1997;22:2807-12. 